\title{
Studying lipoprotein trafficking in zebrafish, the case of chylomicron retention disease
}

\author{
Amnon Schlegel \\ Published online: 10 January 2015 \\ (C) Springer-Verlag Berlin Heidelberg 2015
}

The gastro-intestinal system digests, absorbs, and then transports dietary nutrients. The small intestine is the major site of all three processes, where luminal contents - amino acids, sugars, fatty acids, cholesterol, and an assortment of minerals and micronutrients - are taken up by enterocytes, packaged and secreted into the circulation. Transport of neutral fats poses a unique problem in that the cargo is not water soluble. These dietary constituents are suspended in micelles within the lumen of the intestine through the surfactant action of bile; luminal lipases then liberate free fatty acids and cholesterol that can traverse the apical surface of the enterocytes (Fig. 1). Following absorption, an evolutionarily conserved machinery resynthesizes neutral lipids for incorporation into chylomicrons, lipoprotein particles that are secreted across the basolateral surface of the enterocyte for delivery to the circulation where they are suspended for transport to sites of lipid delivery [1].

Three autosomal recessive Mendelian causes of defective intestinal fat transport have been described (Fig. 1). To varying degrees, each Mendelian disease of intestinal fat transport is marked by malnutrition, stunting of growth, fat-soluble vitamin deficiencies, and accumulation of fat in the intestine (intestinal lipidosis) and in the liver (hepatic steatosis). The first two diseases abetalipoproteinemia (OMIM: 200100) and

\section{A. Schlegel $(\square)$}

University of Utah Molecular Medicine Program, School of Medicine, University of Utah, 15 North 2030 East, Room 3420B, Salt Lake City, UT 84112, USA

e-mail: amnons@u2m2.utah.edu

\section{A. Schlegel}

Department of Internal Medicine, Division of Endocrinology, Metabolism and Diabetes, School of Medicine, University of Utah, 15 North 2030 East, Room 3420B, Salt Lake City, UT 84112, USA

A. Schlegel

Department of Biochemistry, School of Medicine, University of Utah, 15 North 2030 East, Room 3420B, Salt Lake City, UT 84112, USA hypobetalipoproteinemia (OMIM: 107730) cause defective assembly of nascent chylomicrons owing to mutations in the genes MICROSOMAL TRIGLYCERIDE TRANSFER PROTEIN $(M T T P)$, encoding the chylomicron packaging enzyme, and APOLIPOPROTEIN B $(A P O B)$, encoding the signature apolipoprotein of chylomicrons, respectively. The third single-gene disease in this category is chylomicron retention disease (CMRD; OMIM: 246700, also called Anderson disease), and the affected gene $S A R 1 B$ encodes a guanosine triphosphatase that participates in the coat protein II (COPII)-coated vesicular transport of pre-chylomicrons from the endoplasmic reticulum to the Golgi apparatus [2]. In CMRD, initiation of chylomicron production is normal, but maturation and transport across the enterocyte's secretory system is impaired. From the initial positional cloning of the CMRD locus [3] and numerous subsequent reports, we have learned that nearly all the $S A R 1 B$ lesions identified are point mutations or small insertion-deletions that inactivate the encoded protein's function. Like the rest of the machinery of chylomicron assembly, SAR1B is an ancient and highly conserved protein found in all eukaryotes [4]. Why loss of this protein's function causes a seemingly limited defect in enterocyte lipid transport is a fundamental question that has been stymied by lack of a robust animal model.

In this issue, Knapik and colleagues describe a zebrafish larval model of CMRD [5]. This organism has several technical advantages for studying lipid metabolism, with numerous validated tools for such investigation in both the larval and adult periods [6]. The authors first establish that sarlb messenger RNA (mRNA) is deposited maternally in oocytes. Over the course of the embryonic and larval period, robust sar $1 b$ expression becomes restricted to the intestine and head structures. The authors use translation initiation-disrupting antisense morpholino oligonucleotides (MOs) to knock down expression of the Sarlb protein. They confirm the MO reagent's activity, and show knockdown can be rescued through introduction of MOinsensitive mRNAs encoding both zebrafish Sarlb and human SAR1B proteins. The authors establish that sarlb MO-injected 
Fig. 1 Enterocyte trafficking of dietary lipids. Dietary lipids are suspended in micelles comprised of surfactant bile acids. The triacylglycerol (TAG) and cholesteryl esters (CE) are hydrolyzed into fatty acids (FA) and cholesterol (C), which are absorbed across the apical surface. $\mathrm{C}$ and plant sterols (PS) are carried by the NPC1L1 transmembrane protein into the enterocyte, and plant sterols are immediately excreted by the ABCG5/ABCG8 heterodimer. $\mathrm{FA}$ and $\mathrm{C}$ are re-esterified into TAG and CE at the surface of the endoplasmic reticulum (ER) and incorporated into nascent chylomicrons through the MTPcatalyzed lipidation of ApoB. Prechylomicrons are loaded into COPII-coated pre-chylomicron transport vesicles for delivery to the Golgi, where they mature further before secretion across the basolateral surface for delivery to the lymphatic circulation. Three Mendelian diseases of intestinal lipid transport have been reported. Two involve inactivating mutations of the genes encoding ApoB and MTP, while the third inactivates the guanosine triphosphatase Sar1b, which regulates the trafficking of pre-chylomicrons from the ER. In all three diseases, cytoplasmic lipid droplets accumulate

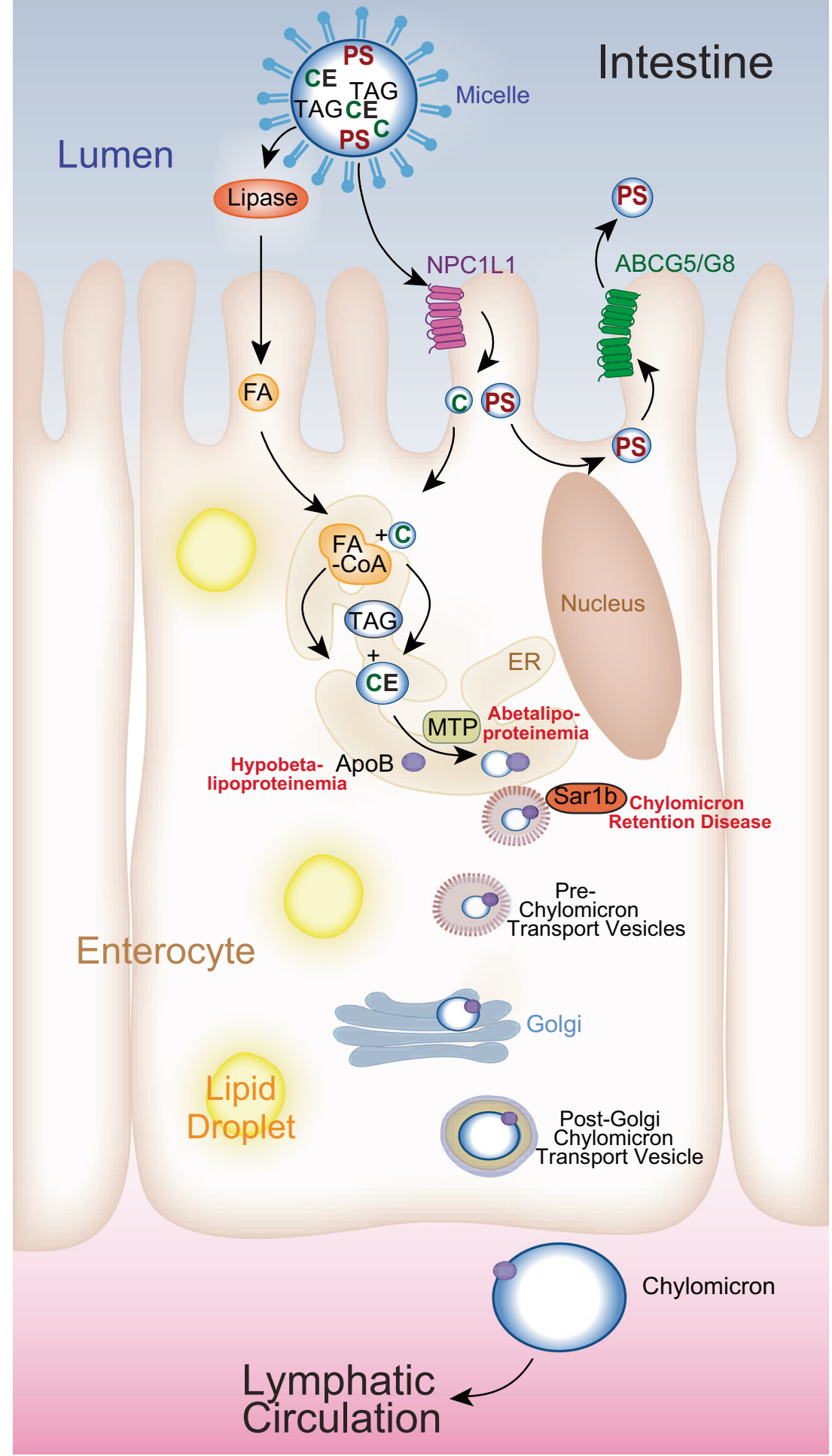

larvae develop intestinal lipidosis when fed a high-fat and highcholesterol meal, with numerous cytoplasmic lipid droplets accumulating in otherwise normal-appearing enterocytes.

The zebrafish sar $1 b$ knockdown model differs from human CMRD in one major respect: the rapidity of the growth defects. In addition to the intestinal transport defect, the authors note decreased endoderm-derived organ size and altered neural development in the MO-injected larvae within the short experimental window of the first week of life. This seeming acceleration of developmental arrest may reflect difference between the rapid embryonic growth of externally fertilized zebrafish embryos and the slower rate of growth 
experienced by placenta-fed human embryos. While the $s a r 1 b$ transcript does not appear to be strongly expressed in the yolk syncytial layer (YSL), the Sarlb protein is very likely to be present in this lipid-transporting cell type: an enhancer trap reporter construct inserted close to the 3'-end of the sarlb gene is expressed in the YSL [7]. Low-level protein expression of Sarlb in the YSL, very likely, explains the seemingly normal use of yolk lipids in sarlb MO-injected animals.

The severe growth retardation seen in sarlb MO-injected larvae is reminiscent of the severe phenotype of zebrafish larvae injected with $m t p$ MOs (the zebrafish ortholog of human MTTP) [8]. The $m t p$ MO-injected embryos also manifest defective absorption of maternally deposited yolk lipids; moreover, this defect appears more severe than the phenotype of a subsequently reported hypomorphic point mutation in $m t p[8,9]$. The $m t p$ mutant has a very interesting vascular patterning defect, reflecting altered lipoprotein-directed signaling to the endothelium [9]. These differences between strong MO-based knockdown phenotypes and chemical mutagen-induced hypomorphic alleles underscore the value of establishing more than one genetic model.

CMRD has a broad clinical spectrum, with neurological manifestations being the most variable [10]. Since the primary sequence of SAR1B proteins are highly conserved from plants to humans, I anticipate the authors will be able to make excellent use of new genome editing technologies to introduce human point mutations into the zebrafish $s a r 1 b$ locus to model the spectrum of CMRD phenotypes [11]. Conversely, they should be able to assess whether constitutive overactivity of Sarb1 causes any changes in chylomicron trafficking. Such knock-ins may allow the authors to perform juvenile and adult experiments, including more detailed physiological measurements of intestinal lipid transport, measurement of plasma lipids, and tracking the fate of radiotracer analogs of dietary lipids [12]. Such experiments might explain why a fluorescent cholesterol analog showed defective uptake by the intestines of the sar $1 b$ MO-injected larvae. Since this cholesterol analog is absorbed only in the presence of luminal fats [13], the author's finding raises the possibility that intestinal Sarlb deficiency triggers alterations in gene expression that limit further cholesterol transport. The simplest molecular basis would be down-regulation of expression of the apical cholesterol transporter Npc111 (Fig. 1). In cultured hepatocytes, Sarlb knockdown causes a coordinated down-regulation of cholesterol synthetic genes, suggesting a parallel method to limit accumulation of free cholesterol in the setting of defective very low-density lipoprotein secretion [14].

Additionally, zebrafish Sarlb knock-in mutant models would allow comprehensive comparison of lipid trafficking gene expression between the intestine and liver. Indeed, the sarlb MO-injected larvae do not display hepatic steatosis, suggesting this phenotype takes longer to develop than the experimental window afforded by these transient knockdown reagents. Lastly, genetic zebrafish CMRD models would afford the opportunity to perform live imaging of lipid transport among organs, something that poses a major technical challenge in other organisms. Assessing how the very large prechylomicrons ( $250 \mathrm{~nm}$ in diameter) are "stuffed" into a COPII vesicle could be tackled with live imaging approaches, as could subsequent maturation of these particles to their final ( $>1-\mu \mathrm{m}$ diameter) size. Loss-of-function studies on additional individual components of the COPII complex would address these questions directly $[15,16]$. Collectively, modeling CMRD in zebrafish will yield insights into enterocyte lipid handling that could be of use not only to understand this rare and potentially devastating monogenetic illness but also to allow investigators to identify novel therapeutic targets for combatting pandemic obesity through modulating enterocyte transport of absorbed nutrients.

\section{References}

1. Peretti N, Sassolas A, Roy C, Deslandres C, Charcosset M, Castagnetti J, Pugnet-Chardon L, Moulin P, Labarge S, Bouthillier $\mathrm{L}$ et al (2010) Guidelines for the diagnosis and management of chylomicron retention disease based on a review of the literature and the experience of two centers. Orphanet J Rare Dis 5:24

2. Kuehn MJ, Herrmann JM, Schekman R (1998) COPII-cargo interactions direct protein sorting into ER-derived transport vesicles. Nature 391:187-190

3. Jones B, Jones EL, Bonney SA, Patel HN, Mensenkamp AR, Eichenbaum-Voline S, Rudling M, Myrdal U, Annesi G, Naik S et al (2003) Mutations in a Sarl GTPase of COPII vesicles are associated with lipid absorption disorders. Nat Genet 34:29-31

4. Gillon AD, Latham CF, Miller EA (2012) Vesicle-mediated ER export of proteins and lipids. Biochim Biophys Acta 1821: 1040-1049

5. Levic DS, Minkel JR, Wang W-D, Rybski WM, Melville DB, Knapik EW (2015) Animal model of Sarlb deficiency presents lipid absorption deficits similar to Anderson disease. J Mol Med (this issue)

6. Schlegel A, Gut P (2015) Metabolic insights from zebrafish genetics, physiology and chemical biology. Cell Mol Life Sci In Press

7. Davison JM, Akitake CM, Goll MG, Rhee JM, Gosse N, Baier H, Halpern ME, Leach SD, Parsons MJ (2007) Transactivation from Gal4-VP16 transgenic insertions for tissue-specific cell labeling and ablation in zebrafish. Dev Biol 304:811-824

8. Schlegel A, Stainier DY (2006) Microsomal triglyceride transfer protein is required for yolk lipid utilization and absorption of dietary lipids in zebrafish larvae. Biochemistry 45:15179-15187

9. Avraham-Davidi I, Ely Y, Pham VN, Castranova D, Grunspan M, Malkinson G, Gibbs-Bar L, Mayseless O, Allmog G, Lo B et al (2012) ApoB-containing lipoproteins regulate angiogenesis by modulating expression of VEGF receptor 1. Nat Med 18:967-973

10. Georges A, Bonneau J, Bonnefont-Rousselot D, Champigneulle J, Rabès J, Abifadel M, Aparicio T, Guenedet J, Bruckert E, Boileau C et al (2011) Molecular analysis and intestinal expression of SAR1 genes and proteins in Anderson's disease (chylomicron retention disease). Orphanet J Rare Dis 6:1-16

11. Peng Y, Clark KJ, Campbell JM, Panetta MR, Guo Y, Ekker SC (2014) Making designer mutants in model organisms. Development 141:4042-4054 
12. Cruz-Garcia L, Schlegel A (2014) Lxr-driven enterocyte lipid droplet formation delays transport of ingested lipids. J Lipid Res 55:19441958

13. Walters JW, Anderson JL, Bittman R, Pack M, Farber SA (2012) Visualization of lipid metabolism in the zebrafish intestine reveals a relationship between NPC1L1-mediated cholesterol uptake and dietary fatty acid. Chem Biol 19:913-925

14. Fryer LGD, Jones B, Duncan EJ, Hutchison CE, Ozkan T, Williams PA, Alder O, Nieuwdorp M, Townley AK, Mensenkamp AR et al (2014) The endoplasmic reticulum coat protein II transport machinery coordinates cellular lipid secretion and cholesterol biosynthesis. J Biol Chem 289:4244-4261

15. Jin L, Pahuja KB, Wickliffe KE, Gorur A, Baumgartel C, Schekman $\mathrm{R}$, Rape M (2012) Ubiquitin-dependent regulation of COPII coat size and function. Nature 482:495-500

16. Butkinaree C, Guo L, Ramkhelawon B, Wanschel A, Brodsky JL, Moore KJ, Fisher EA (2013) A regulator of secretory vesicle size, Kelch-like protein 12, facilitates the secretion of apolipoprotein B100 and very-low-density lipoproteins. Arterioscler Thromb Vasc Biol 34:251-254 